IRSTI 27.41 .41

\author{
${ }^{1}$ S. Aisagaliev, ${ }^{1}$ Zh. Zhunussova, ${ }^{2} \mathrm{H}$. Akca \\ ${ }^{1}$ Al-Farabi Kazakh National University, Almaty, Kazakhstan \\ e-mail: Serikbai.Aisagaliev@kaznu.kz \\ ${ }^{2}$ Abu-Dhabi University, Abu-Dhabi, UAE \\ e-mail: Haydar.Akca@adu.ac.ae

\section{Immersion principle for a variation calculus problem with boundary conditions}

\begin{abstract}
The immersion principle is based on the investigation of the Fredholm integral equation of the first kind. For the Fredholm integral equation of the first kind, the existence theorem for the solution as well as the theorem on its general solution are proved. The basis of the proposed method for solving the variation problem is the immersion principle. The essence of the immersion principle is that the original variation problem with the boundary conditions with phase and integral constraints is replaced by equivalent optimal control problem with a free right end of the trajectory. This approach is made possible by finding the general solution of a class of Fredholm integral equations of the first order. In this work a method for solving the Lagrange problem with phase restrictions for processes described by ordinary differential equations without involvement of the Lagrange principle is supposed. Necessary and sufficient conditions for existence of a solution of the variation problem are obtained, feasible control is found and optimal solution is constructed by narrowing the field of feasible controls. In contrast to the well-known method for solving the problem of the variation calculus on the basis of the Lagrange principle, an entirely new approach an "immersion principle" is proposed.

Key words: immersion principle, feasible control, integral equations, optimal control, optimal solution, minimizing sequence.
\end{abstract}

\section{Problem statement}

We consider the following problem: minimize the functional

$$
\begin{gathered}
J\left(u(\cdot), x_{0}, x_{1}\right)= \\
=\int_{t_{0}}^{t_{1}} F_{0}\left(x(t), u(t), x_{0}, x_{1}, t\right) d t \rightarrow \inf
\end{gathered}
$$

at conditions

$$
\dot{x}=A(t) x+B(t) f(x, u, t), t \in I=\left[t_{0}, t_{1}\right]
$$

with boundary conditions

$$
\left.\left(x\left(t_{0}\right)\right)=x_{0}, x\left(t_{1}\right)=x_{1}\right) \in S_{0} \times S_{1}=S \subset R^{2 n}
$$

in the presence of phase constraints

$$
\begin{gathered}
x(t) \in G(t): G(t)= \\
=\left\{x \in R^{n} / \omega(t) \leq F(x, t) \leq \phi(t), t \in I\right\},
\end{gathered}
$$

and integral constraints

$$
\begin{gathered}
g_{j}\left(u(\cdot), x_{0}, x_{1}\right) \leq 0, \\
j=\overline{1, m_{1}} ; \quad g_{j}\left(u(\cdot), x_{0}, x_{1}\right)=0, \\
j=\overline{m_{1}+1, m_{2}}, \\
g_{j}\left(u(\cdot), x_{0}, x_{1}\right)= \\
=\int_{t_{0}}^{t_{1}} f_{0 j}\left(x(t), u(t), x_{0}, x_{1}, t\right) d t, j=\overline{1, m_{2}},
\end{gathered}
$$


where the control

$$
u(\cdot) \in L_{2}\left(I, R^{m}\right)
$$

Here $A(t), B(t)$ are matrices with piecewisecontinuous elements of orders $n \times n, \quad n \times r$, respectively, a vector function $f(x, u, t)=\left(f_{1}(x, u, t), \ldots, f_{r}(x, u, t)\right)$ continuous with respect to the variables $(x, u, t) \in R^{n} \times R^{m} \times I, \quad$ satisfies the Lipschitz condition by $x$, i.e.

$$
\begin{gathered}
|f(x, u, t)-f(y, u, t)| \leq l(t)|x-y|, \\
\forall(x, u, t),(y, u, t) \in R^{n} \times R^{m} \times I
\end{gathered}
$$

and the condition

$$
\begin{gathered}
|f(x, u, t)| \leq c_{0}\left(|x|+|u|^{2}\right)+c_{1}(t), \\
\forall(x, u, t),
\end{gathered}
$$

where $l(t) \geq 0, \quad l(t) \in L_{1}\left(I, R^{1}\right), \quad c_{0}=$ const $>0$, $c_{1}(t) \geq 0, c_{1}(t) \in L_{1}\left(I, R^{1}\right)$.

The vector function $F(x, t)=\left(F_{1}(x, t), \ldots, F_{s}(x, t)\right)$ is continuous with respect to the variables $(x, t) \in R^{n} \times I$. Function $f_{0}\left(x, u, x_{0}, x_{1}, t\right)=\left(f_{01}\left(x, u, x_{0}, x_{1}, t\right), \ldots\right.$ $\left.\ldots, f_{0 m_{2}}\left(x, u, x_{0}, x_{1}, t\right)\right)$ satisfies the condition

$$
\begin{gathered}
\left|f_{0}\left(x, u, x_{0}, x_{1}, t\right)\right| \leq c_{2}\left(|x|+|u|^{2}+\right. \\
\left.+\left|x_{0}\right|+\left|x_{1}\right|\right)+c_{3}(t), \\
\forall\left(x, u, x_{0}, x_{1}, t\right),\left(y, u, x_{0}, x_{1}, t\right) \in \\
\in R^{n} \times R^{m} \times R^{n} \times R^{n} \times I, \\
c_{2}=\text { const } \geq 0, c_{3}(t) \geq 0, c_{3}(t) \in L_{1}\left(I, R^{1}\right) .
\end{gathered}
$$

Scalar function $F_{0}\left(x, u, x_{0}, x_{1}, t\right)$ is defined and continuous with respect to the variables together with partial derivatives by variables $\left(x, u, x_{0}, x_{1}\right)$, $\omega(t), \varphi(t), \quad t \in I$ are given $s$ - dimensional functions. $S$ is given bounded convex closed set of $R^{2 n}$, the time moments $t_{0}, t_{1}$ are fixed.
In $\quad \begin{aligned} & \text { particular, } \\ & S=\left\{\left(x_{0}, x_{1}\right) \in R^{2 n} / H_{j}\left(x_{0}, x_{1}\right) \leq 0,\right.\end{aligned} \quad j=\overline{1, p_{1}} ;$
$\left.<a_{j},\left(x_{0}, x_{1}\right)>=0, \quad j=\overline{p_{1}+1, p_{2}}\right\}, \quad$ where $H_{j}\left(x_{0}, x_{1}\right), \quad j=\overline{1, p_{1}}$ are convex functions, $a_{j} \in R^{2 n}, j=\overline{p_{1}+1, p_{2}}$ are given vectors.

Note, that if the conditions (1.7), (1.8) are satisfied for any $\operatorname{control} u(\cdot) \in L_{2}\left(I, R^{m}\right)$ and the initial condition $x\left(t_{0}\right)=x_{0}$ of the differential equation (1.2) has a unique solution $x(t), t \in I$. This solution has derivative $\dot{x} \in L_{2}\left(I, R^{n}\right)$ and satisfies equation (1.2) for almost all $t \in I$.

It should be noted that integral constraints

$$
\begin{gathered}
g_{j}\left(u(\cdot), x_{0}, x_{1}\right)= \\
=\int_{t_{0}}^{t_{1}} f_{0 j}\left(x(t), u(t), x_{0}, x_{1}, t\right) d t \leq 0, \\
j=\overline{1, m_{1}},
\end{gathered}
$$

by introducing additional variables $d_{j} \geq 0$, $j=\overline{1, m_{1}}$, can be written in the form

$$
g_{j}\left(u(\cdot), x_{0}, x_{1}\right)=-d_{j}, j=\overline{1, m_{1}} .
$$

$$
\begin{aligned}
& \text { Let } \quad \text { the } \quad \begin{array}{c}
\text { vector } \\
\bar{c}=\left(-d_{1}, \ldots,-d_{m_{1}}, 0,0, \ldots, 0\right) \in R^{m_{2}}, \quad \text { be } \\
\text { where }
\end{array} \\
& d_{j} \geq 0, \quad j=\overline{1, m_{1}} . \quad \text { Let a set } \quad \text { be } \\
& Q=\left\{\bar{c} \in R^{m_{2}} / d_{j} \geq 0, j=\overline{1, m_{1}}\right\}, \text { where } d_{j} \geq 0, \\
& j=\overline{1, m_{1}} \text { are unknown numbers. }
\end{aligned}
$$

Definition 1.1. The triple $\left(u_{*}(t), x_{0}^{*}, x_{1}^{*}\right) \in U \times S_{0} \times S_{1}$ is called by admissible control for the problem (1.1) - (1.6), if the boundary problem (1.2) - (1.6) has a solution. A set of all admissible controls is denoted by $\Sigma$, $\Sigma \subset U \times S_{0} \times S_{1}$.

From this definition it follows that for each element of the set $\Sigma$ the following properties are satisfied: 1$)$ the solutions $x_{*}(t), t \in I$ of the differential equation (1.2), issuing from the point $x_{0}^{*} \in S_{0}$, satisfy the condition $x_{*}\left(t_{1}\right)=x_{1}^{*} \in S_{1}$, and 
also $\left(x_{0}^{*}, x_{1}^{*}\right) \in S_{0} \times S_{1}=S ;$ 2) the inclusion $x_{*}(t) \in G(t), t \in I$ holds; 3 ) for each element of the set $\Sigma$ we have the equality $g\left(u(\cdot), x_{0}, x_{1}\right)=\bar{c}$, where $g\left(u_{*}(\cdot), x_{0}^{*}, x_{1}^{*}\right)=\left(g_{1}\left(u_{*}(\cdot), x_{0}^{*}, x_{1}^{*}\right), \ldots, g_{m_{2}}\left(u_{*}(\cdot), x_{0}^{*}, x_{1}^{*}\right)\right)$.

The following problems are set:

Problem 1.2. Find the necessary and sufficient conditions for the existence of a solution of the boundary value problem (1.2) - (1.6).

Note, that the optimal control problem (1.1) (1.6) has a solution if and only if the boundary value problem (1.2) - (1.6) has a solution.

Problem 1.3. Find an admissible control $\left(u_{*}(t), x_{0}^{*}, x_{1}^{*}\right) \in \Sigma \subset U \times S_{0} \times S_{1}$.

If problem 1 has a solution, then there exists an admissible control.

Problem 1.4. Find the optimal control

$\overline{u_{*}}(t) \in U(t)$, the point $\left(\bar{x}_{0}^{*}, \bar{x}_{1}^{*}\right) \in S_{0} \times S_{1}=S$ and the optimal trajectory $\bar{x}_{*}\left(t ; t_{0}, \bar{x}_{0}^{*}\right), t \in I$,

where $\overline{x_{*}}(t) \in G(t), \quad t \in I, \overline{x_{*}}\left(t_{1}\right)=\overline{x_{1}} \in S_{1}$,

$g_{j}\left(\overline{u_{*}}(\cdot), \bar{x}_{0}^{*}, \bar{x}_{1}^{*}\right) \leq 0, \quad j=\overline{1, m_{1}}$,

$g_{j}\left(\overline{u_{*}}(\cdot), \bar{x}_{0}^{*}, \bar{x}_{1}^{*}\right)=0, \quad j=\overline{m_{1}+1, m_{2}}$,

$J\left(\overline{u_{*}}(\cdot), \bar{x}_{0}^{*}, \bar{x}_{1}^{*}\right)=\inf J\left(\bar{u}(\cdot), \bar{x}_{0}, \bar{x}_{1}\right)$,

$\forall\left(\bar{u}(\cdot), \bar{x}_{0}, \bar{x}_{1}\right) \in L_{2}\left(I, R^{m}\right) \times S_{0} \times S_{1}$.

One of the methods for solving the problem of variation calculus is the Lagrange principle. The Lagrange principle allows to reduce the solution of the original problem to the search for an extremum of the Lagrange functional obtained by introducing auxiliary variables (Lagrange multipliers).

In the classical variation calculus, it is assumed that the solution of the differential equation (1.2) belongs to the space $C^{1}\left(I, R^{n}\right)$ and the control $u(t), t$ $\in I$ of the space $C\left(I, R^{m}\right)$ in the optimal control problems [5], the solution $x \in K C^{l}\left(I, R^{n}\right)$ and control $u(t) \in K C^{l}\left(I, R^{m}\right)$. In this paper, the control $u(t), t \in I$ is chosen from $L_{2}\left(I, R^{m}\right)$, and the solution $x(t), t \in I$ is an absolutely continuous function on the interval $I=\left[t_{0}, t_{1}\right]$. For this case, the existence and uniqueness of the solutions of the initial problem for equation (1.2) are presented in the references $[4,6$, $7,8]$.

The purpose of this paper is to create a method for solving the problem of the variation calculus for processes described by ordinary differential equations with phase and integral constraints that differ from the known methods based on the Lagrange principle. It is a continuation of the scientific research presented in $[9,10]$.

\section{The immersion principle}

Let the vector function be $f_{0}\left(x, u, x_{0}, x_{1}, t\right)=$ $=\left(f_{01}\left(x, u, x_{0}, x_{1}, t\right), \ldots, f_{0 m_{2}}\left(x, u, x_{0}, x_{1}, t\right)\right) . \quad \mathrm{We}$ introduce the vector function $\boldsymbol{\eta}(t)=\left(\boldsymbol{\eta}_{1}(t), \ldots, \boldsymbol{\eta}_{m_{2}}(t)\right)$, $t \in I$ as $\eta(t)=\int_{t_{0}}^{t} f_{0}\left(x(\tau), u(\tau), x_{0}, x_{1}, \tau\right) d \tau, t \in I$.

It follows that

$$
\begin{gathered}
\dot{\eta}(t)=f_{0}\left(x(t), u(t), x_{0}, x_{1}, t\right), \\
\eta\left(t_{0}\right)=0, \eta\left(t_{1}\right)= \\
\bar{c} \in Q,\left(x_{0}, x_{1}\right) \in S, u(t) \in L_{2}\left(I, R^{m}\right), \\
x(t) \in G(t) .
\end{gathered}
$$

Now the optimal control problem $(1.1)-(1.6)$ is written in the form: minimize the functional

$$
\begin{gathered}
J\left(u(\cdot), x_{0}, x_{1}\right)= \\
=\int_{t_{0}}^{t_{1}} F_{0}\left(x(t), u(t), x_{0}, x_{1}, t\right) d t \rightarrow \inf
\end{gathered}
$$

at conditions

$$
\begin{gathered}
\dot{x}=A(t) x+B(t) f(x, u, t), t \in I, \\
\dot{\eta}(t)=f_{0}\left(x(t), u(t), x_{0}, x_{1}, t\right), t \in I, \\
\left(x_{0}, x_{1}\right) \in S=S_{0} \times S_{1}, \\
\eta\left(t_{0}\right)=0, \eta\left(t_{1}\right)=\bar{c} \in Q, \\
x(t) \in G(t), u(\cdot) \in L_{2}\left(I, R^{m}\right), t \in I .
\end{gathered}
$$

Note, that the problems $(1.1)-(1.6)$ and (2.1) (2.5) are equivalent. We introduce the following vectors and matrices

$$
\boldsymbol{\xi}=\left(\begin{array}{l}
x \\
\eta
\end{array}\right), A_{1}(t)=\left(\begin{array}{ll}
A(t) & O_{n, m_{2}} \\
O_{m_{2}, n} & O_{m_{2}, m_{2}}
\end{array}\right)
$$




$$
\begin{gathered}
B_{1}(t)=\left(\begin{array}{c}
B(t) \\
O_{m_{2}, r}
\end{array}\right), B_{2}=\left(\begin{array}{c}
O_{n, m_{2}} \\
I_{m_{2}}
\end{array}\right), \\
\boldsymbol{\xi}\left(t_{0}\right)=\left(\begin{array}{c}
x\left(t_{0}\right)=x_{0} \\
\eta\left(t_{0}\right)=0
\end{array}\right)=\boldsymbol{\xi}_{0}, \boldsymbol{\xi}\left(t_{1}\right)=\left(\begin{array}{c}
x\left(t_{1}\right)=x_{1} \\
\eta\left(t_{1}\right)=\bar{c}
\end{array}\right), \\
P_{1}=\left(I_{n}, O_{n, m_{2}}\right),
\end{gathered}
$$

where $O_{k, q}$ is a rectangular matrix of order $k \times q$ with zero elements, $I_{n}$ is the unit matrix of order $n \times n$.

Then the optimal control problem (2.1) - (2.5) has the form: minimize the functional

$$
\begin{gathered}
J\left(u(\cdot), x_{0}, x_{1}\right)= \\
=\int_{t_{0}}^{t_{1}} F_{0}\left(P_{1} \xi(t), u(t), x_{0}, x_{1}, t\right) d t \rightarrow \inf
\end{gathered}
$$

at conditions

$$
\begin{gathered}
\dot{\boldsymbol{\xi}}=A_{1}(t) \xi+B_{1}(t) f\left(P_{1} \xi, u, t\right)+ \\
+B_{2} f_{0}\left(P_{1} \xi, u, x_{0}, x_{1}, t\right), \\
\xi\left(t_{0}\right)=\xi_{0} \in S_{0} \times O_{m_{2}, 1}, \quad \xi\left(t_{1}\right)=\xi_{1} \in S_{1} \times Q, \\
P_{1} \xi(t) \in G(t), u(\cdot) \in L_{2}\left(I, R^{m}\right), \bar{c} \in Q .
\end{gathered}
$$

Let a set be

$$
\Gamma=\left\{d \in R^{m_{1}} / d \geq 0\right\} .
$$

We consider a linear controllable system

$$
\begin{gathered}
\dot{y}=A_{1}(t) y+B_{1}(t) w_{1}(t)+B_{2} w_{2}(t), t \in I, \\
w_{1}(\cdot) \in L_{2}\left(I, R^{r}\right), w_{2}(\cdot) \in L_{2}\left(I, R^{m_{2}}\right), \\
y\left(t_{0}\right)=\xi\left(t_{0}\right)=\xi_{0} \in S_{0} \times O_{m_{2}, 1}, \\
y\left(t_{1}\right)=\xi\left(t_{1}\right)=\xi_{1} \in S_{1} \times Q .
\end{gathered}
$$

The bases of the immersion principle are the following theorems on the properties of the solution of the Fredholm integral equation of the first kind

$$
K u=\int_{t_{0}}^{t_{1}} K\left(t_{0}, t\right) u(t) d t=a
$$

where $K: L_{2}\left(I, R^{k}\right) \rightarrow R^{n_{1}}, \quad K\left(t_{0}, t\right)$ is a given matrix of order $n_{1} \times k$ with piecewise-continuous elements by $t$ for each fixed $t_{0}, t_{0} \in \Delta_{0} \subset R^{1}$, $t_{1} \in \Delta_{1} \subset R^{1}, \Delta_{0} \cap \Delta_{1}=\varnothing, \varnothing$ is an empty set, $a \in R^{n_{1}}$ is any given vector, $u(\cdot) \in L_{2}\left(I, R^{k}\right)$ is the origin function.

Theorem 2.1. The integral equation (2.14) for any fixed a $a \in R^{n_{1}}$ has a solution if and only if the matrix

$$
C\left(t_{0}, t_{1}\right)=\int_{t_{0}}^{t_{1}} K\left(t_{0}, t\right) K^{*}\left(t_{0}, t\right) d t
$$

of order $n_{1} \times n_{1}$ is positive definite, where $(*)$ is a transposition sign.

Theorem 2.2. Let the matrix $C\left(t_{0}, t_{1}\right)$ be positive definite. Then the general solution of the integral equation (2.14) has the form

$$
\begin{gathered}
u(t)=K^{*}\left(t_{0}, t\right) C^{-1}\left(t_{0}, t_{1}\right) a+v(t)- \\
-K^{*}\left(t_{0}, t\right) C^{-1}\left(t_{0}, t_{1}\right) \int_{t_{0}}^{t_{1}} K\left(t_{0}, t\right) v(t) d t, t \in I,
\end{gathered}
$$

where $v(\cdot) \in L_{2}\left(I, R^{k}\right)$ is an arbitrary function, $a \in R^{n_{1}}$ is any vector.

The proofs of Theorems 2.1. and 2.2. are given in $[9,10]$.

Let the matrix $B_{3}(t)=\left(B_{1}(t), B_{2}\right)$ of order $\left(n+m_{2}\right) \times\left(m_{2}+r\right)$, and a vector function $w(t)=\left(w_{1}(t), w_{2}(t)\right) \in L_{2}\left(I, R^{r+m_{2}}\right)$. It is easy to verify that the control $w(\cdot) \in L_{2}\left(I, R^{r+m_{2}}\right)$, which takes the trajectory of the system (2.9) from any initial state $\xi_{0}$ to any desired final state $\xi_{1}$, is a solution of the integral equation

$$
\int_{t_{0}}^{t_{1}} \Phi\left(t_{0}, t\right) B_{3}(t) w(t) d t=a
$$


where $\Phi(t, \tau)=\lambda(t) \lambda^{-1}(\tau), \lambda(t)$ is a fundamental matrix of solutions of linear homogeneous system $\dot{\rho}=A_{1}(t) \rho$, the vector

$$
a=a\left(\xi_{0}, \xi_{1}\right)=\Phi\left(t_{0}, t_{1}\right) \xi_{1}-\xi_{0} .
$$

As follows from (2.14), (2.17), the matrix $K\left(t_{0}, t\right)=\Phi\left(t_{0}, t\right) B_{3}(t), \quad$ at $\quad n_{1}=n+m_{2}$, $k=r+m_{2}$. For the integral equation (2.15) the statements of Theorems 2.1. and 2.2. are applicable. From the initial data of the system $(2.11)-(2.13)$ we define the following matrices and vectors

$$
\begin{aligned}
& T\left(t_{0}, t_{1}\right)=\int_{t_{0}}^{t_{1}} \Phi\left(t_{0}, t\right) B_{3}(t) B_{3}^{*}(t) \Phi^{*}\left(t_{0}, t\right) d t= \\
& =\int_{t_{0}}^{t_{1}} K\left(t_{0}, t\right) K^{*}\left(t_{0}, t\right) d t=C\left(t_{0}, t_{1}\right), \\
& \Lambda_{1}\left(t, \boldsymbol{\xi}_{0}, \boldsymbol{\xi}_{1}\right)=B_{3}^{*} \Phi^{*}\left(t_{0}, t\right) T^{-1}\left(t_{0}, t_{1}\right) a= \\
& =K^{*}\left(t_{0}, t_{1}\right) C^{-1}\left(t_{0}, t_{1}\right) a= \\
& =\left(\begin{array}{c}
B_{1}^{*}(t) \Phi^{*}\left(t_{0}, t\right) T^{-1}\left(t_{0}, t_{1}\right) a \\
B_{2}^{*} \Phi^{*}\left(t_{0}, t\right) T^{-1}\left(t_{0}, t_{1}\right) a
\end{array}\right), \\
& N_{1}(t)=-B_{3}^{*}(t) \Phi^{*}\left(t_{0}, t\right) T^{-1}\left(t_{0}, t_{1}\right) \Phi\left(t_{0}, t_{1}\right)= \\
& =-K^{*}\left(t_{0}, t\right) C\left(t_{0}, t_{1}\right) \Phi\left(t_{0}, t_{1}\right)= \\
& =\left(\begin{array}{c}
-B_{1}^{*}(t) \Phi^{*}\left(t_{0}, t\right) T^{-1}\left(t_{0}, t_{1}\right) \Phi\left(t_{0}, t_{1}\right) \\
-B_{2}^{*} \Phi^{*}\left(t_{0}, t\right) T^{-1}\left(t_{0}, t_{1}\right) \Phi\left(t_{0}, t_{1}\right)
\end{array}\right)=\left(\begin{array}{c}
N_{11}(t) \\
N_{12}(t)
\end{array}\right), \\
& \Lambda_{2}\left(t, \xi_{0}, \xi_{1}\right)=\Phi\left(t, t_{0}\right) T\left(t, t_{1}\right) T^{-1}\left(t_{0}, t_{1}\right) \boldsymbol{\xi}_{0}+ \\
& +\boldsymbol{\Phi}\left(t, t_{0}\right) T\left(t_{0}, t\right) T^{-1}\left(t_{0}, t_{1}\right) \Phi\left(t_{0}, t_{1}\right) \boldsymbol{\xi}_{1}, \\
& N_{2}(t)=-\Phi\left(t, t_{0}\right) T\left(t_{0}, t\right) T^{-1}\left(t_{0}, t_{1}\right) \Phi\left(t_{0}, t_{1}\right), \\
& t \in T \text {, } \\
& T\left(t, t_{1}\right)=\int_{t}^{t_{1}} \Phi\left(t_{0}, \tau\right) B_{3}(\tau) B_{3}^{*}(\tau) \Phi^{*}\left(t_{0}, \tau\right) d \tau, \\
& T\left(t_{0}, t\right)=T\left(t_{0}, t_{1}\right)-T\left(t, t_{1}\right), t \in I,
\end{aligned}
$$

where the vector a is defined by formula (2.16).
Theorem 2.3. Let the matrix be $T\left(t_{0}, t_{1}\right)>0$.

Then the control

$w(\cdot)=\left(w_{1}(\cdot), w_{2}(\cdot)\right) \in L_{2}\left(I, R^{r+m_{2}}\right)$ transforms the trajectory of the system (2.11) - (2.13) from the initial point $\xi_{0} \in S_{0} \times O_{m_{2}, 1}$ to the final state $\xi_{1} \in S_{1} \times Q$ if and only if

$$
\begin{gathered}
w_{1}(t) \in W_{1}=\left\{w_{1}(\cdot) \in L_{2}\left(I, R^{r}\right) / w_{1}(t)=\right. \\
=v_{1}(t)+B_{1}^{*}(t) \Phi^{*}\left(t_{0}, t\right) T^{-1}\left(t_{0}, t_{1}\right) a+ \\
+N_{11}(t) z\left(t_{1}, v\right),
\end{gathered}
$$

$$
\begin{aligned}
t \in I, \quad \forall & \left.v_{1}(\cdot), v_{1}(\cdot) \in L_{2}\left(I, R^{r}\right)\right\}, \\
w_{2}(t) \in W_{2}= & \left\{w_{2}(\cdot) \in L_{2}\left(I, R^{m_{2}}\right) / w_{2}(t)=\right. \\
=v_{2}(t)+B_{2}^{*} \Phi^{*}\left(t_{0}, t\right) T^{-1}\left(t_{0}, t_{1}\right) a+ & \\
& +N_{12}(t) z\left(t_{1}, v\right),
\end{aligned}
$$

$$
\left.t \in I, \forall v_{2}(\cdot), v_{2}(\cdot) \in L_{2}\left(I, R^{m_{2}}\right)\right\},
$$

where $\quad v(t)=\left(v_{1}(t), v_{2}(t)\right), \quad v_{1}(\cdot) \in L_{2}\left(I, R^{r}\right)$, $v_{2}(\cdot) \in L_{2}\left(I, R^{m_{2}}\right)$ are arbitrary functions. The function $z(t, v)=z\left(t, v_{1}, v_{2}\right), t \in I$ is the solution of the differential equation

$$
\begin{gathered}
\dot{z}=A_{1} z+B_{1}(t) v_{1}(t)+B_{2} v_{2}(t), \\
z\left(t_{0}\right)=0, t \in I, \\
v_{1}(\cdot) \in L_{2}\left(I, R^{r}\right), v_{2}(\cdot) \in L_{2}\left(I, R^{m_{2}} .\right.
\end{gathered}
$$

The solution of the differential equation (2.11) corresponding to the control (2.19), (2.20) has the form

$$
\begin{gathered}
y(t)=z(t)+\Lambda_{2}\left(t, \boldsymbol{\xi}_{0}, \boldsymbol{\xi}_{1}\right)+N_{2}(t) z\left(t_{1}, v\right), \\
t \in I,
\end{gathered}
$$

where $z(t)=z\left(t_{1}, v\right), t \in I$. 
Proof. The proof of the theorem follows from Theorems 2.1. and 2.2. As follows from the above, the solution of the boundary value problem (2.11) (2.13) reduces to finding the general solution of the integral equation (2.17). The integral equation (2.17) is a particular case of (2.14), where $K\left(t_{0}, t\right)=\Phi\left(t_{0}, t\right) B_{3}(t)$. Further, by replacing $K\left(t_{0}, t\right) \quad$ on $\Phi\left(t_{0}, t\right) B_{3}(t) \quad$ we get $C\left(t_{0}, t_{1}\right)=T\left(t_{0}, t_{1}\right) \quad$ (see (2.15)). From (2.16) follows (2.19), (2.20). The differential equation (2.21) with control (2.22) and relation (2.23) follows directly from formulas

$$
\begin{gathered}
z(t, v)=\int_{t_{0}}^{t} \Phi(t, \tau) B_{3}(\tau) v(\tau) d \tau \\
z\left(t_{1}, v\right)=\Phi\left(t_{1}, t_{0}\right) \int_{t_{0}}^{t_{1}} \Phi\left(t_{0}, t\right) B_{3}(t) v(t) d t
\end{gathered}
$$

It is easy to see, that $y\left(t_{0}\right)=\xi_{0}, y\left(t_{1}\right)=\xi_{1}$. The theorem is proved.

Note, that: 1) the sets $W_{1}=W_{1}(t) \subset L_{2}\left(I, R^{r}\right)$, $W_{2}=W_{2}(t) \subset L_{2}\left(I, R^{m_{2}}\right)$ contain all sets of the functions $w_{1}(t), w_{2}(t), \quad t \in I$, for which the boundary value problem $(2.11)-(2.13)$ has a solution; 2) $w_{1}(t) \in W_{1}, \quad w_{2}(t) \in W_{2}$, then the solution of the system (2.11) - (2.13) is defined by formula (2.23); 3) outside sets $W_{1}, W_{2}$ there are no controls for which the boundary value problem (2.11) - (2.13) has a solution; 4) Theorem 2.3. allows to replace the boundary value problem (2.11) - (2.13) by the initial problem (2.21) - (2.23).

Lemma 2.4. Let the matrix be $T\left(t_{0}, t_{1}\right)>0$. Then the boundary problem (2.7) - (2.10) is equivalent to the following problem

$$
\begin{gathered}
w_{1}(t) \in W_{1}, w_{1}(t)=f\left(P_{1} y(t), u(t), x_{0}, x_{1}, t\right), \\
t \in I, \\
w_{2}(t) \in W_{2}, w_{2}(t)=f_{0}\left(P_{1} y(t), u(t), x_{0}, x_{1}, t\right), \\
t \in I,
\end{gathered}
$$

$$
\begin{gathered}
p(t)=F\left(P_{1} y(t), t\right) \in V=V(t)=\{p(\cdot) \in \\
\left.\in L_{2}\left(I, R^{s}\right) / \omega(t) \leq p(t) \leq \phi(t), t \in I\right\}, \\
\dot{z}=A_{1}(t) z+B_{1}(t) v_{1}(t)+ \\
+B_{2} v_{2}(t), z\left(t_{0}\right)=0, t \in I, \\
v_{1}(\cdot) \in L_{2}\left(I, R^{r}\right), v_{2}(\cdot) \in L_{2}\left(I, R^{m_{2}}\right), \\
\left(x_{0}, x_{1}\right) \in S_{0} \times S_{1}=S \subset R^{2 n}, \\
u(\cdot) \in L_{2}\left(I, R^{m}\right), d \in \Gamma,
\end{gathered}
$$

where the function $y(t), t \in I$ is defined by the formula (2.23).

Proof. Lemma 2.4. states, that the boundary value problem $(2.7)-(2.10)$ has a solution if and only if the relations $(2.24)-(2.29)$ are satisfied.

In fact, if the relations (2.24) - (2.29) are held, then $y(t)=\xi(t), \quad t \in I, \quad$ moreover $y\left(t_{0}\right)=\xi\left(t_{0}\right)=\xi_{0}, \quad y\left(t_{1}\right)=\xi\left(t_{1}\right)=\xi_{1} \quad$ and the inclusions (2.9), (2.10) are satisfied.

We suppose, that the boundary value problem $(2.7)-(2.10)$ has a solution. It is possible if and only if $f\left(P_{1} \xi(t), u(t), t\right) \in W_{1}$, $f_{0}\left(P_{1} \xi(t), u(t), x_{0}, x_{1}, t\right) \in W_{2}$ by Theorem 2.3. These inclusions are equivalent to equalities (2.24), (2.25), where $z(t), t \in I-$ is a solution of the differential equation (2.27) with controls (2.28). The inclusion $P_{1} \xi(t) \in G(t), t \in I$ has the form (2.26), and the relations (2.9), (2.10) are written in the form (2.29). The lemma is proved.

Lemma 2.5. Let the matrix be $T\left(t_{0}, t_{1}\right)>0$. Then the boundary value problem of optimal control with constraints (1.1) - (1.6) is equivalent to the following problem: minimize the functional

$$
\begin{aligned}
& I\left(u(\cdot), p(\cdot), v_{1}(\cdot), v_{2}(\cdot), x_{0}, x_{1}, d\right)= \\
= & \int_{t_{0}}^{t_{1}} F_{0}\left(P_{1} y(t), u(t), x_{0}, x_{1}, t\right) d t \rightarrow \inf
\end{aligned}
$$

at conditions 


$$
\begin{gathered}
I_{1}\left(u(\cdot), p(\cdot), v_{1}(\cdot), v_{2}(\cdot), x_{0}, x_{1}, d\right)= \\
=\int_{t_{0}}^{t_{1}} F_{1}(q(t), t) d t=\int_{t_{0}}^{t_{1}}\left[\mid w_{1}(t)-\right. \\
-\left.f\left(P_{1} y(t), u(t), t\right)\right|^{2}+\mid w_{2}(t)- \\
-\left.f_{0}\left(P_{1} y(t), u(t), x_{0}, x_{1}, t\right)\right|^{2}+ \\
\left.+\left|p(t)-F\left(P_{1} y(t), t\right)\right|^{2}\right] d t=0, \\
\dot{z}=A_{1}(t) z+B_{1}(t) v_{1}(t)+B_{2} v_{2}(t), \\
z z\left(t_{0}\right)=0, t \in I, \\
v_{1}(\cdot) \in L_{2}\left(I, R^{r}\right), v_{2}(\cdot) \in L_{2}\left(I, R^{m_{2}}\right), \\
\left(x_{0}, x_{1}\right) \in S_{0} \times S_{1}=S \subset R^{2 n}, \\
p(t) \in V(t), u(\cdot) \in L_{2}\left(I, R^{m}\right), d \in \Gamma,
\end{gathered}
$$

where $w_{1}(t) \in W_{1}, w_{2}(t) \in W_{2}$, the function $y(t)$, $t \in I$ is defined by the formula (2.23).

Proof. The proof follows from Lemma 2.4. The value of the functional $I_{1} \geq 0$ The functional $I_{1}=0$ if and only if the equalities (2.24) - (2.26) are satisfied, relations (2.27) - (2.29) coincide with (2.31) - (2.33). The functional (1.1) can be written in the form (2.30). The lemma is proved.

The function

$$
\begin{gathered}
F_{1}(q(t), t)= \\
=\left|w_{1}(t)-f\left(P_{1} y(t), u(t), x_{0}, x_{1}, t\right)\right|^{2}+ \\
+\left|w_{2}(t)-f_{0}\left(P_{1} y(t), u(t), x_{0}, x_{1}, t\right)\right|^{2}+ \\
+\left|p(t)-F\left(P_{1} y(t), t\right)\right|^{2},
\end{gathered}
$$

where $w_{1}(t) \in W_{1}, w_{2}(t) \in W_{2}$,

$y(t)=z(t, v)+\Lambda_{2}\left(t, \xi_{0}, \xi_{1}\right)+N_{2}(t) z\left(t_{1}, v\right), t \in I$, $q(t)=\left(z(t, v), z\left(t_{1}, v\right), u(t), p(t), v_{1}(t), v_{2}(t), x_{0}, x_{1}, d\right)$

Note, that:

1) Since the initial problem (1.1) - (1.6) is equivalent to $(2.30)-(2.33)$, that the problem (1.1) - (1.6) has a solution if and only if the relations $(2.30)-(2.33)$ are satisfied;
2) Since the value $I_{1} \geq 0$, that for the existence of a solution of the boundary value problem (1.2) (1.6) it is necessary and sufficient that inf $I_{1}\left(u, p, v_{1}, v_{2}, x_{0}, x_{1}, d\right)=0 \quad$ under the conditions $(2.27)-(2.29)$.

3) The transition from the original boundary value problem (1.2) - (1.6) to the initial optimal control problem $I_{1}\left(u, p, v_{1}, v_{2}, x_{0}, x_{1}, d\right) \rightarrow$ inf under the conditions $(2.27)-(2.29)$ is called the immersion principle.

\section{Conclusion}

The Lagrange problem of the variation calculus is investigated in the presence of phase and integral constraints for processes described by ordinary differential equations. The particular cases of which are the simplest problem, the Bolz problem, the isoperimetric problem, the conditional extremum problem.

The main scientific results are:

-reduction of the boundary value problem connected to the conditions in the Lagrange problem to the initial optimal control problem with a specific functional;

- necessary and sufficient conditions for the existence of the admissible control;

- method of constructing an admissible control on the limit point of the minimizing sequence;

The scientific novelty of the results is that: there is no need to introduce additional variables in the form of Lagrange multipliers; proof of the existence of a saddle point of the Lagrange functional.

\section{References}

1. Bliss Dzh. "Lectures on variation calculus." (M.: IL, 1950), 348.

2. Lavrentev M.A., Lyusternik L.A. "Basics of variation calculus." ONTI 1 (1935): 148-400.

3. Yang L. "Lectures on variation calculus and optimal control theory." Mir (1974): 488.

4. Alekseev V.M., Tihomirov V.M., Fomin S.V. “Optimal control." Nauka (1979): 430.

5. Pontryagin L.S., Boltyanskiy V.G., Gamkrelidze R.V., Mischenko E.F. "Mathematical theory of optimal processes." Nauka (1969): 384. 
6. Varga Dzh. "Optimal control by differential and functional equations." Nauka (1977): 624.

7. Gamkrelidze R.V. "Basics of optimal control.” (Tbilisi: Izd-vo Tbilisskogo n-ta, 1977), 253.

8. Li Ya.B., Markus L. "Basics of the optimal control theory." Nauka (1972): 574.
9. Aysagaliev S.A., Sevryugin I.V. "Controlabillity and speed of the process described by linear system of ordinary differential equations." Mathematical journal 13, no. 2(4) (2013): 5-30.

10. Aysagaliev S.A., Kabidoldanova A.A. "Optimal control by dynamic systems." Palmarium Academic Publishing (Verlag, Germany, 2012): 288. 